\title{
Impact of High Serum Levels of MMP-7, MMP-9, TGF- $\beta$ and PDGF Macrophage Activation Markers on Severity of COVID-19 in Obese-Diabetic Patients
}

\author{
Asmaa Nasr El-Din' \\ Kamal Abd El-Sattar Ata ${ }^{2}$ \\ Abdelhady Ragab Abdel- \\ Gawad (1D ${ }^{3}$ \\ Nahed F Fahmy (D) \\ 'Microbiology and Immunology \\ Department, Faculty of Medicine, Sohag \\ University, Sohag, Egypt; ${ }^{2}$ Chest Diseases \\ and Tuberculosis Department, Faculty of \\ Medicine, Sohag University, Sohag, Egypt; \\ ${ }^{3}$ Department of Clinical and Chemical \\ Pathology, Faculty of Medicine, Sohag \\ University, Sohag, Egypt
}

\begin{abstract}
Objective: The aim of this study was to identify an association between the severity of COVID-19 in obese-diabetic patients and altered serum levels of MMP-7, MMP-9, TGF- $\beta$, and PDGF macrophage activation markers.

Methodology: The study included 70 COVID-19 patients, divided into two groups: Group 1 included: Obese COVID-19 patients with type 2 diabetes mellitus (T2D, $n=22$ patients) and group 2 included; non-obese, non-diabetic COVID-19 patients as an age- and sex-matched control group ( $\mathrm{n}=48$ patients). Serum levels of the tested biomarkers were measured by ELISA at admission and after one weak follow-up.

Results: There was a significant reduction in the serum levels of LBP in obese-diabetic COVID-19 patients versus the control group (8.34 \pm 3.94 vs 20.78 \pm 7.61 ) (p 0.0001). Significant elevation of MMP-7, MMP-9, PDGF and TGF- $\beta$ was detected in obese diabetic COVID-19 patients compared to the non-obese non-diabetic group: $1044.7 \pm 519.6$ vs 405.6 $\pm 164.1,483.05 \pm 46.5$ vs $173.31 \pm 76.26,154.5 \pm 62.78$ vs $39.77 \pm 21.52$, and $603.05 \pm 258.82$ vs $180.29 \pm 97.17$, respectively. The serum levels of macrophage activation markers in obesediabetic patients one week after admission revealed that patients with acute respiratory distress syndrome (ARDS) had significantly higher serum levels of MMP-7 and MMP-9 than non-ARDS patients (p 0.02 and p 0.01 respectively).

Conclusion: Macrophages were mainly polarized towards the M2 phenotype in obesediabetic COVID-19 patients with significant upregulation of the pro-fibrotic markers MMP-7, MMP-9, PDGF, and TGF- $\beta$. Thus, high levels of MMP-7 and MMP-9 are associated with ARDS in severe COVID-19 disease among obese-diabetic patients.
\end{abstract}

Keywords: COVID-19, TGF- $\beta$, PDGF, MMP-7, MMP-9

\section{Introduction}

Coronaviruses are enveloped viruses containing a single-stranded positive sense RNA genome enclosed in a helical nucleocapsid. Coronaviruses produce diseases in human with variable severity. Of the seven known human coronaviruses (HCos), four $\mathrm{HCos}$ cause symptoms of the common cold in humans including ( $\mathrm{HCoV}$ OC43, HCoV-HKU1, HCoV-229E, HCoV-NL63), and three HCos can cause more serious diseases and include severe acute respiratory syndrome (SARS) caused by SARS-CoV, Middle East respiratory syndrome (MERS) caused by MERS-CoV, ${ }^{1}$ and COVID-19 (COronaVIrus Disease 2019), which is a pandemic
Correspondence: Asmaa Nasr El-Din Email asmaanasreldin8I@gmail.com 
disease caused by a novel coronavirus known as Severe Acute Respiratory Syndrome Coronavirus 2 (SARSCoV-2). ${ }^{2}$

According to the clinical presentation, COVID-19 can be classified into three phases. In Phase I, viral replication occurs. It is benign and self-limited and patients may be asymptomatic or present with flu-like manifestations in the form of fever, dry cough, fatigue, sore throat, loss of taste and smell and sometimes patients present with gastrointestinal manifestations. Spontaneous resolution occurs in $80 \%$ of patients without complications. Phase II, or the hyper-inflammatory phase, occurs in $15 \%$ of infected patients who manifest acute respiratory distress syndrome (ARDS). These patients require intensive care unit admission and ventilator-support. ${ }^{3}$ Phase III, which occurs in 5\% of patients, it is characterized by a cytokine storm, which is an inflammatory immune response leading to organ failure and development of microthrombi or pulmonary emboli due to hypercoagulability. ${ }^{4}$ The disease severity in COVID-19 patients is not only due to viral infection but is also due to the host immune response. ${ }^{5}$

SARS-CoV-2 entry into the host cells is mediated by a transmembrane spike protein, which mediates binding to the cellular receptor angiotensin-converting enzyme 2 (ACE2). ${ }^{6}$ Infection and replication of the virus within the epithelial cells of the airways result in high levels of viruslinked apoptosis of infected cells, inducing inflammatory responses mediated by pro-inflammatory cytokines and chemokines. Subsequently, recruitment of macrophages and monocytes to the site of infection and activation of $\mathrm{T}$ cells and $\mathrm{B}$ cells result in the resolution of the infection in most cases. However, in some patients, immune dysregulation occurs, with the secretion of aberrant pro-inflammatory cytokines by alveolar macrophages. ${ }^{7}$

Different populations of macrophages, including macrophages recruited from the blood and alveolar macrophages (AMs) resident in the lungs, undergo dramatic changes in the phenotype and ultimately exert an important role in the pathogenesis and resolution of ARDS, the hallmark of severe COVID-19. ARDS is associated with increased mortality especially in diabetic patients. ${ }^{8}$

In response to infection, macrophages undergo polarization and result in the formation of two phenotypes: The classically activated phenotype M1 macrophage is differentiated in response to lipopolysaccharide (LPS), interferon-gamma (IFN- $\gamma$ ) and tumor necrosis factor-alpha (TNF- $\alpha$ ). M1 macrophages are important in virus clearance as they exhibit pro-inflammatory cytotoxic properties. These macrophages express CD69, CD38, Toll-like receptor (TLR) 2 (TLR2), TLR4, CXC-chemokine ligand 9 (CXCL9), CXCL10 and CXCL11. The alternatively activated phenotype M2 macrophage is differentiated in response to Th2-type cytokines, including interleukin (IL)-4 and IL-13, macrophage colony-stimulating factor (MCSF) or TLR ligands, M2 macrophages exert anti-inflammatory properties and play an important role in post-injury mechanisms by mediating matrix deposition and tissue remodeling. M2 macrophages express mannose receptors (CD206), matrix metalloproteinase (MMP) 2 (MMP2), MMP7, MMP9 and CD163. ${ }^{9}$

An increase in the number of tissue macrophages is a prominent feature of micro- and macro-vascular complications of diabetes. Some studies have reported that hyperglycemia suppresses phagocytic ability and increases the expression of M2 macrophage markers, such as MMP9 and CD169. ${ }^{10}$ Chronic activation of the M2 macrophage phenotype leads to the production of pro-fibrotic markers including platelet-derived growth factor (PDGF) and transforming growth factor- $\beta$ (TGF- $\beta$ ) that enhance the activation of fibroblasts and proliferation of myofibroblasts. ${ }^{11}$ In addition, alveolar M2 macrophages produce fibro-proliferative markers, such as MMP7 and MMP9, which promote fibrogenesis in diabetic and obese patients leading to lung stiffening; thus, patients are more vulnerable to COVID-19 infections. ${ }^{12}$ Variability in glucose levels has also been associated with increased tissue damage through oxidative stress and further, increased glucose variability is associated with more severe ARDS in COVID-19 infections. ${ }^{13}$

This study aimed to determine whether the polarization toward M2 macrophage is associated with the severity of COVID-19 in diabetic-obese patients, through measurement of serum levels of M2 macrophage markers and by correlating high levels of these markers to the deteriorating clinical and laboratory findings of patients during followup. To the best of our knowledge, this is the first study in Egypt that has addressed this issue.

\section{Materials and Methods Study Population}

This cross-sectional study was conducted from April 2020 to April 2021 at the Central Research Laboratory and Medical Microbiology and Immunology Department, Faculty of Medicine, Sohag University. Our study included 70 patients who were admitted to the Sohag Teaching Hospital, 
a designated isolation hospital in Sohag, with a definite diagnosis of COVID-19 by real-time PCR. The study population was classified into 2 groups: Group 1 comprised obese COVID-19 patients with type 2 diabetes mellitus (T2D, $\mathrm{n}=22$ patients) and group 2 comprised non-obese, non-diabetic COVID-19 patients as an age- and sex-matched control group ( $\mathrm{n}=48$ patients).

\section{Inclusion Criteria Group I}

COVID-19 patients diagnosed clinically, and by laboratory and radiological findings and confirmed positively by realtime PCR. T2D diagnosed for $<10$ years and all patients were on stable doses of anti-diabetic medications for the preceding three months (eg, metformin, statin and/or angiotensin-converting enzyme inhibitor/angiotensin receptor blocker). Obese patients were defined by a body mass index $(\mathrm{BMI}) \geq 30 \mathrm{~kg} / \mathrm{m} 2$.

\section{Group 2}

COVID-19 patients diagnosed clinically, and by laboratory and radiological findings and confirmed positively by realtime PCR, with no clinical history of T2D or obesity.

\section{Exclusion Criteria}

Patients with any one of the following criteria were excluded from our study: those with a history of smoking, excessive alcohol consumption, HIV infection, neutropenia, chronic chest disease (asthma, bronchiectasis, and pulmonary fibrosis), chronic renal disease, or malignancy.

\section{Diagnosis of COVID 19 Clinically}

In our study, we relied on the guidelines of the National Institutes of Health (NIH) that classify COVID-19 into the following disease types: ${ }^{14}$

\section{Mild Illness}

Patients presenting with fever, cough, malaise, headache, sore throat, muscle pain, nausea, vomiting, diarrhea, anosmia, loss of taste, chills and gastrointestinal manifestations but without shortness of breath or abnormal chest imaging.

\section{Moderate IIIness}

Patients presenting with clinical manifestations or radiologic evidence of lower respiratory tract illness and oxygen saturation $(\mathrm{SpO} 2) \geq 94 \%$ on room air.

\section{Severe Illness}

Patients presenting with $\mathrm{SpO} 2 \leq 94 \%$ on room air; a ratio of partial pressure of arterial oxygen to fraction of inspired oxygen $(\mathrm{PaO} 2 / \mathrm{FiO} 2)<300$ with lung infiltrates $>50 \%$ or marked tachypnea with respiratory rate of $>30$ breaths $/ \mathrm{min}$.

\section{Critical Illness}

Patients presenting with acute respiratory failure, septic shock, and/or multiple organ failure or presented cytokine storm. ARDS tended to occur in severe COVID-19 approximately one week after the onset of symptoms with patients becoming critically ill.

\section{Laboratory Findings}

Molecular Testing was considered the standard method for diagnosis of COVID-19. ${ }^{15}$ Real-time PCR assays on nasopharyngeal swab were used for the detection of SARSCoV-2 nucleic acid.

Altered complete blood counts, defined by lymphopenia, eosinopenia, thrombocytopenia and neutrophil/lymphocyte ratio $\geq 3.13$ were associated with COVID-19 and indicated worse prognosis.

Elevated values of the following parameters were considered as risk factors for severe disease:

C-reactive protein (CRP) (normal values; $0.8-3.0 \mathrm{mg} / \mathrm{L}$ ), D-dimer (reference value $<250 \mathrm{ng} / \mathrm{mL}$ ), and ferritin (30-300 $\mathrm{ng} / \mathrm{mL}$ for males, and $30-160 \mathrm{ng} / \mathrm{mL}$ for females).

\section{Radiological Findings}

(a) Computed tomography (CT) of the chest showed high sensitivity and was based on COVID-19 Reporting and Data System (CO-RADS) which is a chest CT-based system used to assess the pulmonary involvement in COVID-19 patients as was scored as follows: ${ }^{16}$

CO-RADS Category 0 : Technically insufficient examination.

CO-RADS Category 1: No suspicion of COVID-19 infection. The $\mathrm{CT}$ is normal or there are findings that indicate a non-infectious disease.

CO-RADS Category 2: Low level of suspicion of COVID-19 infection. Abnormalities are consistent with infections other than COVID-19.

CO-RADS Category 3: Intermediate level of suspicion of COVID-19 infection. CT abnormalities indicating infection, but unsure whether COVID-19 is involved. 
CO-RADS Category 4: High level of suspicion of COVID-19 infections. Mostly these are suspicious CT findings but not extremely typical; unilateral ground glass, multifocal consolidations without any other typical finding, and/or findings suspicious of COVID-19 in underlying pulmonary disease.

CO-RADS Category 5: Very high level of suspicion of COVID-19 infection. Typical CT findings; Mandatory features are ground-glass opacities, which were bilateral and peripherally distributed in lung regions close to visceral pleural surfaces with or without consolidations (Figure 1).
Confirmatory features:

- Unsharp demarcated and half rounded multiple ground-glass areas, accompanied by sharply demarcated ground-glass areas that outline the shape of multiple adjacent secondary pulmonary lobules.

- A "crazy paving" pattern shows visible intralobular lines.

- Consolidations appear within the ground-glass opacities.

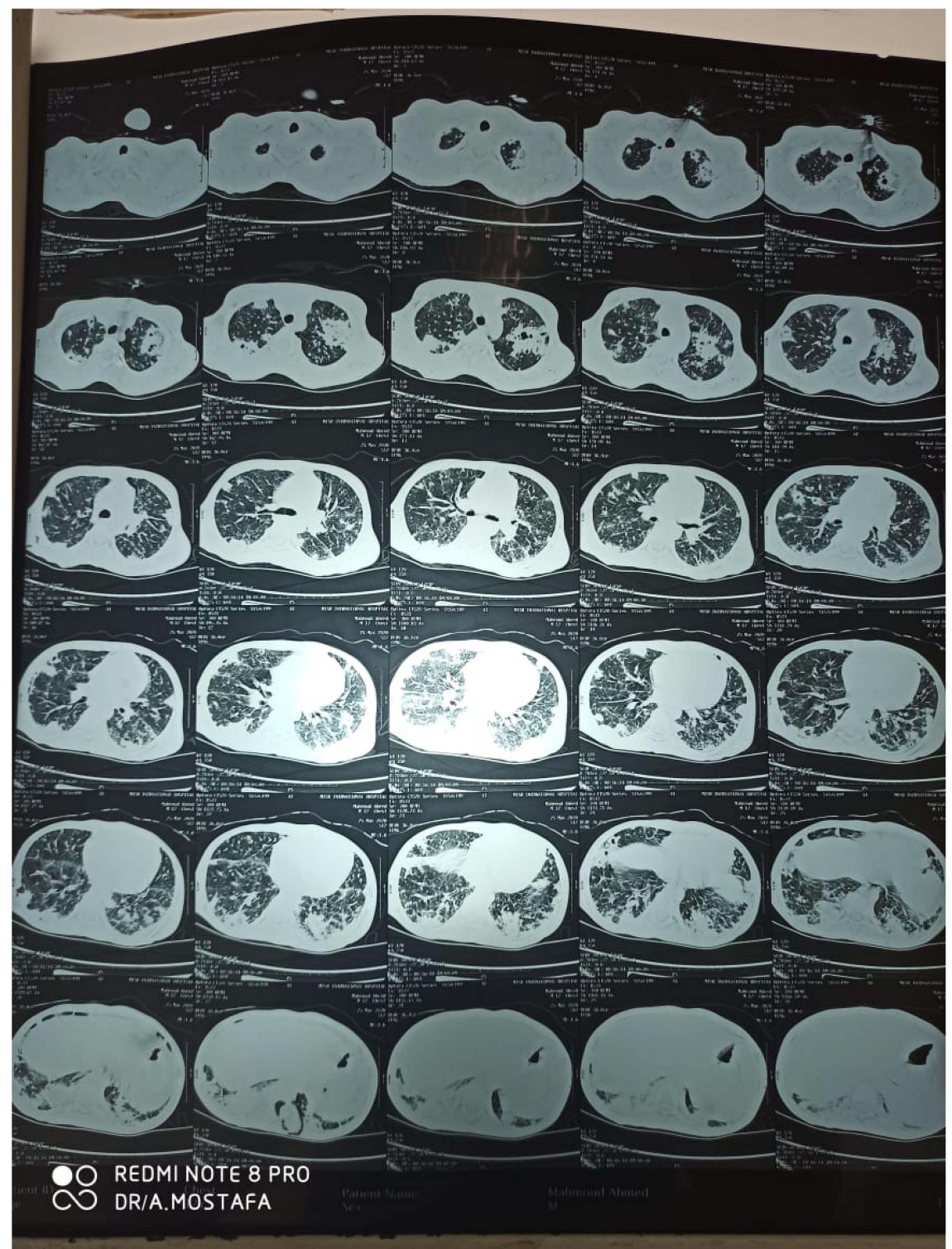

Figure I Variable sized pulmonary and sub-pleural patches of ground glass density and consolidation involving both lungs; impressive of atypical viral pneumonia COVID-I9 CO-RADS 5. 
- "Reverse halo" signs or ground-glass opacity with excessive subpleural consolidations.

- Thickened vessels within lung findings.

CO-RADS Category 6: Confirmed diagnosis of COVID-19 infections by RT-PCR positivity for SARSCoV-2.

(a) Pulmonary ultrasonography showed good sensitivity and characterized by consolidations, pleural thickening and B-lines.

(b) Plain chest X-ray was of low sensitivity and revealed bilateral consolidations accompanied by peripheral ground glass opacities in the lower lobes. $^{32}$

\section{Diagnosis of COVID-19 ARDS}

ARDS diagnosis depended on a set of clinical and ventilator criteria.

- Chest imaging findings: ARDS was characterized by worsening of an already identified respiratory picture or severe new-onset respiratory failure. Chest CT showing bilateral opacities (lung infiltrates $>50 \%$ ), not explained by effusions or lung collapse.

- The Berlin definition was used to classify ARDS into three types based on the degree of hypoxia, the partial pressure of arterial oxygen/fraction of inspired oxygen $\left(\mathrm{PaO}_{2} / \mathrm{FIO}_{2}\right)$. The criteria for the $\mathrm{PaO}_{2} / \mathrm{FIO}_{2}$ ratio were modified for COVID-19 ARDS as follows: Mild-moderate disease between 150 and $200 \mathrm{mmHg}$ and Moderate-severe disease $<150 \mathrm{mmHg}$. ${ }^{17}$

COVID-19 patients in both study groups were evaluated at the day of admission to the isolation hospital for symptoms and signs of ARDS and re-evaluated again after one week.

\section{Quantitative Evaluation of Marker Levels Measurement of Lipopolysaccharide Binding Protein by ELISA}

Sera were collected from COVID-19 patients of both groups for quantitative measurement of lipopol ysaccharide-binding protein (LBP) at the time of admission by ELISA kits (Human lipopolysaccharidebinding protein, Catalog: SG-11142, China) according to the manufacturer's instructions (detection range; $0.7-40 \mathrm{ng} / \mathrm{mL})$.
Measurement of M2 Macrophage Activation Markers (MMP-7, MMP-9, PDGF, TGF- $\beta$ ) by ELISA

M2 macrophage activation markers were measured at the day of hospital admission in the sera of COVID-19 patients of both groups 1 and 2. Measurements of these markers were repeated one week after admission in the sera of the COVID-19 patients in group 1 only. Sera were collected from patients for quantitative measurement by ELISA of the following markers:

- MMP-7 (Human matrix metalloproteinase 7 ELISA Kit, Catalog; SG-10410, detection range; 0.5-20 $\mu \mathrm{g} /$ L, China).

- MMP-9 (Human matrix metalloproteinase 9 ELISA Kit, Catalog; SG-10412, detection range; 40-800 $\mu \mathrm{g} /$ L, China).

- PDGF (Human Platelet-Derived Growth Factor ELISA Kit, Catalog; SG-11323, detection range; 4.6-300 pg/mL, China).

- TGF- $\beta$ (Human Transforming Growth factor $\beta$ ELISA Kit, Catalog SG-10060, detection range; $0.78-50 \mathrm{ng} / \mathrm{mL}$, China). All according to the manufacturer's instructions.

All assays were performed according to the manufacturer's instructions.

\section{Ethical Considerations}

Informed written consent was obtained from all the patients or their first-degree relatives; the study protocol was approved by the local Ethics Committee of Scientific Research of the Faculty of Medicine, Sohag University. The study was retrospectively registered in clinical trial registry (clinical trial.gov ID: NCT04766294) and complied with the specifications Declaration of Helsinki.

\section{Statistical Analysis}

Data were analyzed using STATA version 14.2 (Stata Statistical Software: Release 14.2 College Station, TX: StataCorp LP). Quantitative data was represented as mean, standard deviation, median and range. Data were analyzed using Mann-Whitney test for data that were not normally distributed. Qualitative data were presented as number and percentage and compared using either Chi square test or Fisher's exact test. Graphs were produced using Excel or STATA. A p-value was considered significant if it was $\leq 0.05$. 


\section{Results}

This cross-sectional study was conducted on 70 patients with confirmed diagnosis of COVID-19 who were admitted to the Sohag Teaching Isolation Hospital. The ages of the patients were classified as follows: $26 \%$ of the patients aged from 44 to $<55$ years, $58 \%$ aged from 55 to $<65$ years, and $16 \%$ aged from 65 to 75 years. Overall, $41.2 \%$ of the patients included in the study were males, and $58.8 \%$ were females. By assessment of the severity of COVID-19 in the patients included; $25 \%$ presented mild illness, 62\% moderate illness, and 13\% severe COVID-19. The study group was divided into 2 groups: Group 1 included 22 obese and diabetic COVID-19 patients (31.4\%), and group 2 included 48 non-obese and nondiabetic COVID-19 patients $(68.6 \%)$.

According to the results of the laboratory tests, obesediabetic COVID-19 patients presented higher serum levels of ferritin and D-dimer in comparison to non-obese nondiabetic COVID-19 patients (p-values 0.001 and 0.01 respectively) (Figures 2 and 3). Based on the pulmonary involvement as detected by chest-CT, COVID-19 patients were assigned to different CO-RAD categories: $50 \%$ of obese-diabetic COVID-19 patients were assigned to CORAD category 5 , while only $18.75 \%$ of non-obese nondiabetic COVID-19 patients were assigned to CO-RAD category 5, this difference was significant (p 0.03) (Table 1).

Patients were evaluated for COVID-19 ARDS on the day of hospital admission based on the clinical, ventilatory and chest imaging data. We found no significant

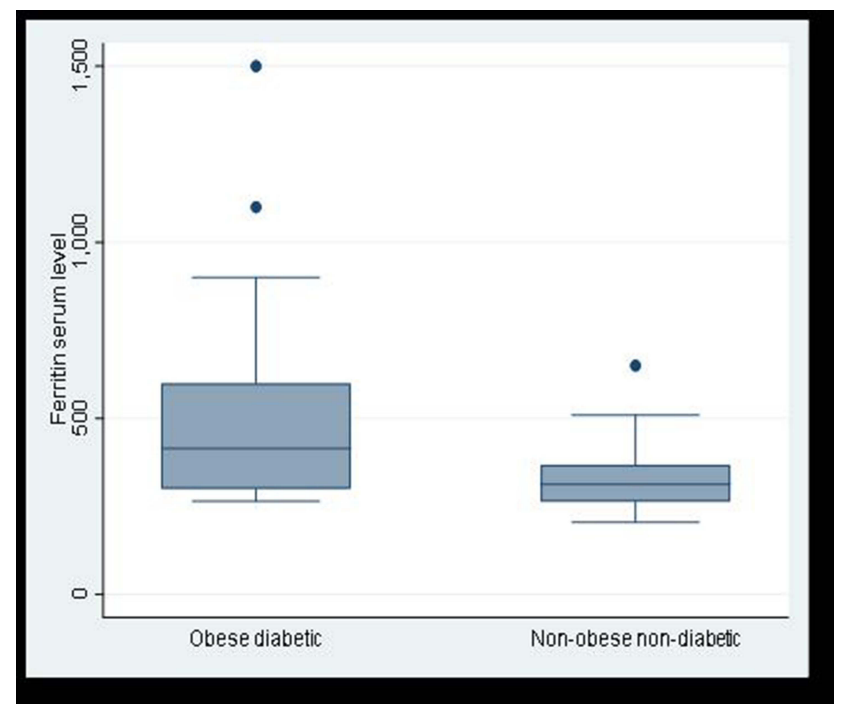

Figure 2 Comparison between obese diabetic patients and non-obese non-diabetic patients as regards serum ferritin level.

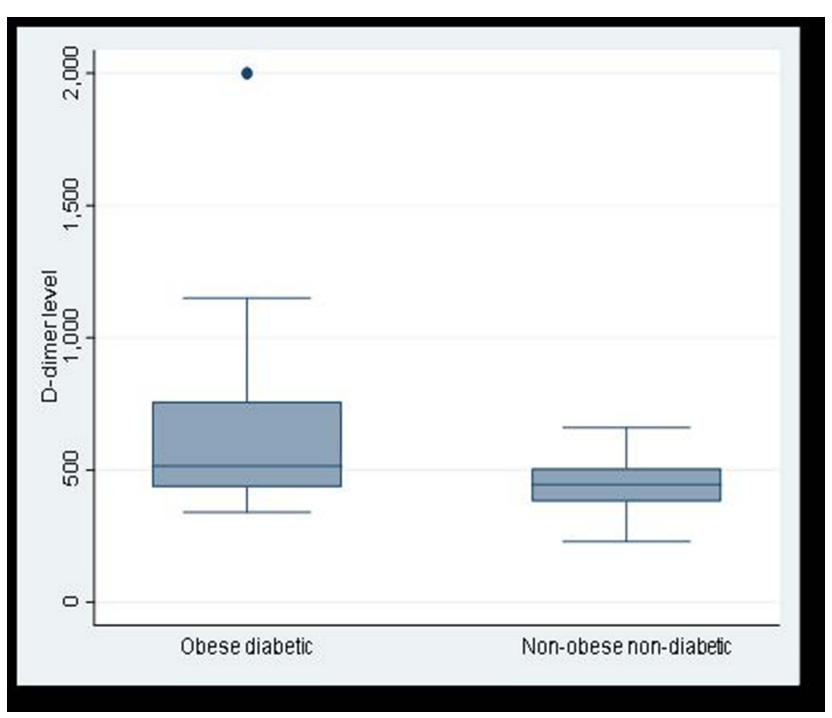

Figure 3 Comparison between obese diabetic patients and non-obese non-diabetic patients as regards D- dimer plasma level.

difference in the frequency of development of ARDS between the 2 study groups (Table 1). While on re-evaluation of patients for the development of ARDS one week after hospital admission, the presence of ARDS was significantly more frequent $(p$-value $=0.03$ ) among obese diabetic COVID-19 patients $(n=8 / 22)$ than the control group $(n=6 / 48)$ (Figure 4$)$.

LBP was measured by ELISA. We detected a significant reduction in the serum levels of LBP in obese-diabetic COVID-19 patients compared to the nonobese non-diabetic group (p 0.0001) (Table 2).

Serum levels of M2 macrophage activation markers were quantitatively measured at the day of hospital admission for all study participants, and the serum levels of these markers were compared between the 2 study groups. We found a significant elevation of MMP-7, MMP-9, PDGF and TGF- $\beta$ levels in obese diabetic COVID-19 patients compared to non-obese non-diabetic group (Table 3).

Obese-diabetic patients were observed for the development of COVID-19 ARDS and accordingly they were divided into 2 subgroups, those who developed ARDS and those who did not. By comparing the levels of macrophage activation markers measured at the day of admission between the 2 subgroups, ARDS patients had significantly higher serum levels of MMP-7 and MMP-9. The serum levels of PDGF and TGF- $\beta$ were higher in the serum of ARDS patients, albeit with no statistical significance (Table 4).

The serum levels of macrophage activation markers were measured in obese diabetic patients one week after 
Table I Laboratory, Radiological, and Clinical Features of Obese-Diabetic COVID-19 Patients versus Non-Obese NonDiabetic Patients

\begin{tabular}{|c|c|c|c|}
\hline Variable & $\begin{array}{c}\text { Obese } \\
\text { Diabetic } \\
\mathrm{N}=\mathbf{2 2}\end{array}$ & $\begin{array}{c}\text { Non-Obese Non- } \\
\text { Diabetic } N=48\end{array}$ & $P$ value \\
\hline $\begin{array}{l}\text { Ferritin serum level (ng/ } \\
\mathbf{m L}) \\
\text { Mean } \pm \text { SD } \\
\text { Median (range) }\end{array}$ & $\begin{array}{l}512.95 \pm 314.05 \\
415(265: 1500)\end{array}$ & $\begin{array}{l}318.83 \pm 80.87 \\
313(205: 650)\end{array}$ & 0.001 \\
\hline $\begin{array}{l}\text { D-dimer level (ng/mL) } \\
\text { Mean } \pm \mathrm{SD} \\
\text { Median (range) }\end{array}$ & $\begin{array}{l}648.23 \pm 375.11 \\
515(341: 2000)\end{array}$ & $\begin{array}{l}456.98 \pm 1 \mid 2.69 \\
445.5(230: 661)\end{array}$ & 0.01 \\
\hline $\begin{array}{l}\text { CO-RADS Category; } \\
\text { CO-RADS Category } 3 \text { CO- } \\
\text { RADS Category } 4 \text { CO- } \\
\text { RADS Category } 5\end{array}$ & $\begin{array}{l}5(22.73 \%) \\
6(27.27 \%) \\
11(50.00 \%)\end{array}$ & $\begin{array}{l}19(39.58 \%) \\
20(41.67 \%) \\
9(18.75 \%)\end{array}$ & 0.03 \\
\hline $\begin{array}{l}\text { ARDS at admission } \\
\text { No } \\
\text { Yes }\end{array}$ & $\begin{array}{c}20 \text { (90.91\%) } \\
2 \text { (9.09\%) }\end{array}$ & $\begin{array}{c}47 \text { ( } 97.92 \%) \\
\text { I (2.08\%) }\end{array}$ & 0.23 \\
\hline
\end{tabular}

admission. Patients with ARDS had higher serum levels of MMP-7 and MMP-9 than non-ARDS patients, and this difference was statistically significant. ARDS patients had higher serum levels of PDGF and TGF- $\beta$, albeit the difference was not statistically significant (Table 5).

\section{Discussion}

SARS-CoV-2 infection leading to severe COVID-19 disease in patients with underlying medical conditions such as diabetes is associated with poor outcomes. ${ }^{18}$ In addition, obesity is a significant, independent risk factor for poor outcomes in COVID-19, as the BMI is positively correlated with increased risk of ARDS. ${ }^{19}$ Therefore, patients with obesity and $\mathrm{T} 2 \mathrm{D}$ are at high risk for severe infection with poor outcomes. ARDS results from an excessive and uncontrolled
Table 2 Levels of LBP in Obese-Diabetic versus Non-Obese Non-Diabetic COVID-19 Patients

\begin{tabular}{|l|c|c|c|}
\hline LBP (ng/mL) & $\begin{array}{c}\text { Obese Diabetic } \\
\mathbf{N = 2 2}\end{array}$ & $\begin{array}{c}\text { Non-Obese Non- } \\
\text { Diabetic N=48 }\end{array}$ & P value \\
\hline Mean \pm SD & $8.34 \pm 3.94$ & $20.78 \pm 7.61$ & $<0.0001$ \\
Median (range) & $7.2(2.8: 19.2)$ & $21.85(12.1: 37.2)$ & \\
\hline
\end{tabular}

Table 3 Serum Levels of MMP-7, MMP-9, PDGF, and TGF- $\beta$ in Obese-Diabetic versus Non-Obese Non-Diabetic COVID-19 Patients

\begin{tabular}{|c|c|c|c|}
\hline $\begin{array}{l}\text { M2 Macrophage } \\
\text { Activation Markers }\end{array}$ & $\begin{array}{c}\text { Obese Diabetic } \\
\mathbf{N}=\mathbf{2 2}\end{array}$ & $\begin{array}{l}\text { Non-Obese Non- } \\
\text { Diabetic N=48 }\end{array}$ & $P$ value \\
\hline $\begin{array}{l}\text { MMP-7 ( } \mu g / L) \\
\text { Mean } \pm \text { SD } \\
\text { Median (range) }\end{array}$ & $\begin{array}{c}1044.77 \pm 519.63 \\
\text { | } 162.5(249: 1917)\end{array}$ & $\begin{array}{l}405.63 \pm 164.19 \\
388.5(90: 699)\end{array}$ & $<0.0001$ \\
\hline $\begin{array}{l}\text { MMP-9 }(\mu \mathrm{g} / \mathrm{L}) \\
\text { Mean } \pm \text { SD } \\
\text { Median (range) }\end{array}$ & $\begin{array}{c}483.05 \pm 464.5 \\
464.5(300: 750)\end{array}$ & $\begin{array}{l}173.31 \pm 76.26 \\
168.5(25: 315)\end{array}$ & $<0.0001$ \\
\hline $\begin{array}{l}\text { PDGF (pg/mL) } \\
\text { Mean } \pm \text { SD } \\
\text { Median (range) }\end{array}$ & $\begin{array}{l}154.5 \pm 62.78 \\
|4|(77: 280)\end{array}$ & $\begin{array}{c}39.77 \pm 21.52 \\
38(7: 83)\end{array}$ & $<0.0001$ \\
\hline $\begin{array}{l}\text { TGF- } \beta \text { ( } \mathrm{ng} / \mathrm{mL} \text { ) } \\
\text { Mean } \pm \text { SD } \\
\text { Median (range) }\end{array}$ & $\begin{array}{l}603.05 \pm 258.82 \\
549(267: 1170)\end{array}$ & $\begin{array}{l}\mid 80.29 \pm 97.17 \\
\mid 44.5(69: 42 \mid)\end{array}$ & $<0.0001$ \\
\hline
\end{tabular}

systemic inflammatory response where macrophages, or resident AMs, undergo dramatic changes in number and phenotype, and play an important role in the pathogenesis of ARDS. ${ }^{20}$ ARDS is the hallmark of severe COVID-19 disease, associated with increased mortality particularly among patients with diabetes.

MMPs belong to a family of zinc-dependent endoproteinases that cleave extracellular matrix proteins (eg, collagen, fibronectin, and elastin), and soluble metabolic

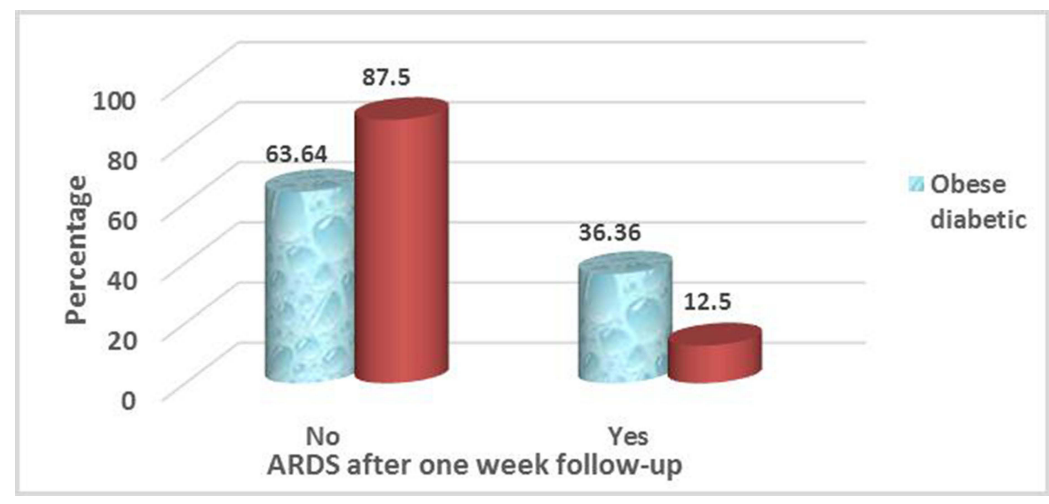

Figure 4 ARDS after one week follow-up in obese diabetic patients versus non-obese non-diabetic patients. 
Table 4 Serum Levels of MMP-7, MMP-9, PDGF, and TGF- $\beta$ on Admission in Obese-Diabetic Patients Who Developed ARDS versus Those Who Did Not Develop ARDS

\begin{tabular}{|l|c|c|c|}
\hline \multirow{2}{*}{ Variable } & \multicolumn{2}{|c|}{ Obese Diabetic COVID-19 Patients } & P value \\
\cline { 2 - 4 } & ARDS N=8 & No ARDS N=14 & \\
\hline MMP-7 at admission & & & 0.03 \\
Mean \pm SD & $1170.17 \pm 583.05$ & $880.36 \pm 544.80$ & \\
Median (range) & $940.5(270: 1917)$ & $850.5(281: 1701)$ & \\
\hline MMP-9 at admission & & & 0.01 \\
Mean \pm SD & $801.67 \pm 155.33$ & $478.93 \pm 134.79$ & \\
Median (range) & $478.5(311: 690)$ & $464.5(300: 750)$ & \\
\hline PDGF at admission & & & 0.59 \\
Mean \pm SD & $162.67 \pm 63.13$ & $148.64 \pm 61.55$ & \\
Median (range) & $156(98: 260)$ & $131.5(77: 280)$ & \\
\hline TGF- $\beta$ at admission & & & 0.41 \\
Mean \pm SD & $672.17 \pm 315.36$ & $558.21 \pm 203.03$ & \\
Median (range) & $660(363: 1170)$ & $549(267: 981)$ & \\
\hline
\end{tabular}

Table 5 Comparison of MMP-7, MMP-9, PDGF, and TGF- $\beta$ Serum Levels Between Obese-Diabetic Patients Who Developed ARDS versus Patients Who Did Not Develop ARDS One Week After Admission

\begin{tabular}{|l|c|c|c|}
\hline \multirow{2}{*}{ Variable } & \multicolumn{2}{|c|}{ Obese Diabetic COVID-19 Patients } & \multirow{2}{*}{ P value } \\
\cline { 2 - 3 } & ARDS N=6 & No ARDS N=14 & \\
\hline MMP-7 at follow-up & & & \multirow{2}{*}{0.02} \\
Mean \pm SD & $2149.36 \pm 1049.91$ & $1295 \pm 1273.98$ & \\
Median (range) & $1895(150: 3140)$ & $835(190: 3150)$ & \\
\hline MMP-9 at follow-up & $811.64 \pm 315.67$ & $481.5 \pm 233.59$ & \multirow{2}{*}{0.01} \\
Mean \pm SD & $520(201: 790)$ & $493.5(190: 743)$ & \\
Median (range) & $187.43 \pm 81.50$ & $144.17 \pm 94.85$ & 0.25 \\
\hline PDGF at follow-up & $165(71: 299)$ & $128.5(40: 288)$ & \\
Mean \pm SD & & & \multirow{2}{*}{0.80} \\
Median (range) & $668.71 \pm 466.04$ & $582.5 \pm 492.63$ & \\
\hline TGF- $\beta$ at follow-up & $493(105: 1401)$ & $419(121: 1500)$ & \\
Mean \pm SD & & & \\
Median (range) & & & \\
\hline
\end{tabular}

mediators. In the lung, MMPs are secreted from inflammatory cells (eg, AMs and neutrophils) and parenchymal cells (eg, pulmonary endothelial cells, interstitial fibroblasts and type II epithelial cells). ${ }^{21}$

In healthy lung tissues, MMPs expression is very limited; MMPs are down-regulated by cytokines as IL-10, and IFN $-\gamma{ }^{22}$ Meanwhile, other members of the MMPs family (MMP-7, MMP-9, and MMP-12) are up-regulated in response to a variety of factors including (i) Transcription factors such as nuclear factor kappa B. (ii)
Growth factors such as epidermal growth factor receptor that up-regulates MMP-9 expression. (iii) Chemokines such as CCL6 that increases MMP-9. (iv) Cytokines such as TNF- $\alpha$, IL-1, IL-6, and IL-13 that increase MMP-2. (v) Endogenous inflammatory intermediates such as reactive oxygen species that activate transcription factors related to MMP gene expression. (vi) Cigarette smoke (which increases MMP-9), and (vii) Mechanical stress including mechanical ventilation. ${ }^{23}$

MMPs play key roles in lung immunity by facilitating the recruitment or regression of inflammatory cells, and modulate the activities of cytokines and chemokines, mediating intercellular signaling, and responses to exogenous stimuli by inducing repair of the pulmonary epithelium. However, the deregulation of the balance between MMPs and their inhibitors, the disturbance of MMP activity, and the excessive secretion of several MMPs can induce inflammatory responses in the respiratory airways, as well as excessive tissue remodeling, and tissue damage. Consequently, MMPs are involved in processes leading to the emergence of acute and chronic lung inflammatory conditions. ${ }^{23}$

Many studies have revealed that MMPs are implicated in lung pathologies. For example, MMP-9 contributes to COPD-associated pulmonary damage, while MMP-7 and MMP-9 are associated with asthma, ARDS and idiopathic pulmonary fibrosis. ${ }^{24}$

In this study, MMP-7 and MMP-9 levels were significantly higher in the serum of obese-diabetic COVID 19 patient group than in the non-obese, non-diabetic group $(\mathrm{p}<0.0001$ for both). Both markers also were significantly elevated in obese diabetic patients who developed ARDS than those who did not develop ARDS during the follow-up period (p 0.02 and $p 0.01$ respectively).

A previous study conducted by Hsu et $\mathrm{al}^{25}$ showed that patients who developed traditional ARDS had a notable increase in plasma MMP-9 activity on a particular day correlated with a decrease in the $\mathrm{PaO}_{2} / \mathrm{FiO}_{2}$ ratio on the following day $(\mathrm{r}=-0.503, \mathrm{p}<0.006)$. These results suggested that plasma MMP-9 had predictive value for the development of traditional ARDS in critically ill patients. ${ }^{25}$

Ueland et $\mathrm{al}^{26}$ measured the serum levels of markers reflecting inflammation, fibrosis and vascular inflammation to determine their association with respiratory function as reflected by the $\mathrm{PaO}_{2} / \mathrm{FiO}_{2}$ ratio in COVID-19 patients. Ueland et al found that only MMP-9 was associated with 
the $\mathrm{PaO}_{2} / \mathrm{FiO}_{2}$ ratio could distinguish patients with and without respiratory failure, suggesting that MMP-9 may be an early indicator of respiratory failure in COVID-19 patients. $^{26}$

Moin et $\mathrm{al}^{12}$ found that MMP7 and MMP9 were elevated in obese T2D COVID-19 patients compared to controls $\mathrm{p}<0.05)$. The authors suggested that enhanced tissue response to SARS-CoV-2 infection was reflected by an increase in the serum levels of MMP-7 and MMP-9 and may predispose diabetics to increased susceptibility to COVID-19 associated ARDS and increased vulnerability for development of severe COVID-19 disease. ${ }^{12}$

LPS is a potential inducer of innate immune response; it promotes an inflammatory cascade by stimulation of the nuclear factor-B pathway resulting in transcription of proinflammatory genes. ${ }^{27}$ Thus, elevated plasma LPS is the key component for activation of M1 macrophages. ${ }^{13}$ LBP, an acute-phase protein synthesized in the liver, initiates recognition of LPS by delivering LPS to the membrane and requires soluble forms of CD14, both finally interact with TLR-4, triggering a signaling cascade that leads to the up-regulation of pro-inflammatory cytokines. ${ }^{28}$

The short half-life of LPS limits the utility of LPS testing. Meanwhile, LBP has a relatively long half-life, and its presence could reflect "effective" LPS levels and the activation of the innate immune response triggered by LPS. $^{29}$

In this study, we measured the serum levels of LBP, which reflect the activity of LPS. We found that the LBP levels were significantly lower in obese diabetic COVID19 patients than in the non-obese non-diabetic group. These results suggest that M1 macrophages are down regulated in obese diabetic patients.

ARDS is a devastating critical illness. ARDS develops in $67 \%$ of COVID-19 patients with the severe illness, and it is considered the main cause of death. ${ }^{30}$ COVID-19 associated ARDS presents some distinguishing features compared with traditional ARDS, including (1) delayed onset of 8-12 days from onset of symptoms, which falls outside the one-week onset of the Berlin ARDS criteria, (2) hyperinflammatory response, and (3) pulmonary microthrombi within the lung vasculature that contribute to ventilation-perfusion mismatch. ${ }^{31}$

In this study, there was no difference in the incidence of ARDS developed on the day of admission between obese diabetic COVID-19 patients versus non-obese nondiabetic patients. However, when patients were re-evaluated for development of ARDS one week after admission, we found that the incidence ARDS was significantly higher among obese-diabetic COVID-19 patients than in non-obese non-diabetic group. Our results were in accordance with the results of $\mathrm{Xu}$ et al $2021^{32}$ who analyzed the risk factors for development of ARDS in COVID-19 patients. The authors found that $52.3 \%$ of ARDS patients had a BMI $>25$, while $31.7 \%$ of non-ARDS patients had a BMI $>25$ (p 0.007). In addition, $17.8 \%$ of ARDS patients were diabetic, while $9.5 \%$ of non-ARDS patients had a history of diabetes ( $\mathrm{p} 0.04$ ).

PDGFs are disulfide-linked hetero-dimeric peptides that act mainly on stromal cells, such as fibroblasts, smooth muscle cells, and pericytes. PDGFs were originally isolated from platelets and are also secreted by monocytes, macrophages, endothelial cells, and fibroblasts. PDGFs play important roles in regulating the differentiation, proliferation, and survival of mesenchymal progenitor cells, and play a vital role in wound healing; however, dysregulated PDGF signaling plays a role in fibrosis. ${ }^{33}$

In this study, the serum levels of PDGF were significantly elevated in obese diabetic patients than non-obese non-diabetic group, while there was a minor increase in the serum levels of PDGF in the ARDS subgroup of obese-diabetic patients, but this difference was not statistically significant. Thus, higher serum levels of PDGF were not considered a direct risk for the development of ARDS in obese diabetic COVID-19 patients.

Petrey et al also ${ }^{34}$ identified growth factors including PDGF and fibroblast growth factor-2 (FGF-2) had a potent role in vessel remodeling and in angiogenesis. These growth factors were significantly increased in the plasma of COVID-19 patients compared to healthy controls. Petrey et al also $^{34}$ observed that there was no significant difference in the plasma levels of these growth factors between moderate and severe COVID-19 patients, confirming circulating levels of these growth factors may be independent of ARDS status.

The major cellular sources of TGF- $\beta$ in pulmonary fibrosis have been shown to be AMs and metaplastic type II alveolar epithelial cells. ${ }^{12}$

In this study, obese-diabetic patients presented significantly higher serum levels of TGF- $\beta$ than non-obese nondiabetic COVID-19 patients, and there was a non-significant minor increase in the ARDS subgroup of obese diabetic patients. Thus, high serum levels of TGF- $\beta$ may not be considered a risk for ARDS in obese diabetic COVID-19 patients. Ferreira-Gomes et $\mathrm{al}^{35}$ found that, in patients with severe COVID-19, SARS-CoV-2 triggers a chronic immune 
reaction that is mediated by TGF- $\beta$, and the authors concluded that the therapeutic targeting of TGF- $\beta$ may be an approach to ameliorate severe COVID-19 symptoms, especially, when considering the fibrosis-inducing capacity of TGF- $\beta .{ }^{35}$ Consistent with our results, Moin et al also reported a significant elevation of basal TGF- $\beta 1$ levels among T2D patients $(1123 \pm 72$ vs $933 \pm 27$, OT2D vs control, $p<0.01){ }^{12}$

The limitations of this study include its small sample size especially among obese-diabetic COVID-19 patients and the limited number of articles describing macrophage activation markers for COVID-19 patients, which are considered an obstacle for comparing data from previous studies with our findings.

\section{Conclusion}

LPS is the main activator of M1 macrophages and is responsible for the inflammatory response. We reported herein the low serum levels of LBP in obese-diabetic COVID-19 patients, and therefore suggest that macrophages were not polarized toward M1 phenotype in these patients. However, macrophages in the lung tissue were more polarized towards the M2 phenotype in obese-diabetic COVID-19 patients with significant upregulation of profibrotic markers MMP-7, MMP-9, PDGF, and TGF- $\beta$. Two matrix metalloproteinases MMP-7 and MMP-9 were significantly elevated in obese diabetic COVID-19 patients who developed ARDS. Our findings indicate that the lung epithelial barrier integrity is destabilized in response to the fibro-proliferative activity of elevated MMP-7 or MMP-9 and may sensitize the alveoli to a pre-fibrotic condition following SARSCoV-2 infection. Thus, we conclude that high levels of MMP-7 and MMP-9 are associated severe COVID-19 disease and ARDS in obese-diabetic patients.

\section{Data Sharing Statement}

The authors agreed and signed not to publish the raw data of the patients of this study; however, the collected and analyzed datasets are available on request from the corresponding author.

\section{Author Contributions}

All authors contributed to data analysis, drafting or revising the article, gave final approval of the version to be published, agreed to the submitted journal, and agree to be accountable for all aspects of the work.

\section{Disclosure}

The authors declare that they have no financial or nonfinancial conflicts of interest related to the work reported in the manuscript. Each author listed in the manuscript saw and approved the submission of this version of the manuscript and takes full responsibility for it. This article had not been published elsewhere and is not currently under consideration by another journal or a publisher.

\section{References}

1. Lu R, Zhao X, Li J, Niu P, Yang B, Wu H. Genomic characterization and epidemiology of 2019 novel coronavirus: implications for virus origins and receptor binding. Lancet. 2020;395(10224):565e574. doi:10.1016/S0140-6736(20)30251-8

2. Guo YR, Qing-Dong C, Hong ZS, et al. The origin, transmission and clinical therapies on Coronavirus disease 2019 (COVID-19) outbreak: an update on the status. Mil Med Res. 2020;7:11.

3. Soares SC, Tenório EA, de Oliveira LH, et al. COVID-19 and obesity: the meeting of two pandemics. Arch Endocrinol Metab. 2021;65(1):1-13.

4. McGonagle D, Sharif K, O'Regan A, Bridgewood C. The role of cytokines including interleukin-6 in COVID-19 induced pneumonia and macrophage activation syndrome-like disease. Autoimmun Rev. 2020;19(6):102537. doi:10.1016/j.autrev.2020.102537

5. Li B, Yang J, Zhao F, et al. Prevalence and impact of cardiovascular metabolic diseases on COVID-19 in China. Clin Res Cardio. 2020;109(5):531-538. doi:10.1007/s00392-020-01626-9

6. Zhou P, Yang XL, Wang XG, Hu B, Zhang L, Zhang W. A pneumonia outbreak associated with a new Coronavirus of probable bat origin. Nature. 2020;579(7798):270-273. doi:10.1038/s41586-020-2012-7

7. Chen $\mathrm{G}, \mathrm{Wu} \mathrm{D}$, Guo W, et al. Clinical and immunological features of severe and moderate Coronavirus disease 2019. J Clin Invest. 2020;13:453-461.

8. Huang I, Lim MA, Pranata R. Diabetes mellitus is associated with increased mortality and severity of disease in COVID-19 pneumonia-A systematic review, meta-analysis, and meta-regression. Diabetes Metab Syndr. 2020;14 (4):395-403. doi:10.1016/j.dsx.2020.04.018

9. Martinez FO, Sica A, Mantovani A, Locati M. Macrophage activation and polarization. Front Biosci. 2008;13(13):453-461. doi: $10.2741 / 2692$

10. Ferracini M, Martins JO, Campos MR, Anger DB, Jancar S. Impaired phagocytosis by alveolar macrophages from diabetic rats is related to the deficient coupling of LTs to the Fc gamma R signaling cascade. Mol Immunol. 2010;47(11-12):1974-1980. doi:10.1016/j.molimm.20 10.04.018

11. Prasse A, Pechkovsky DV, Toews GB, et al. A vicious circle of alveolar macrophages and fibroblasts perpetuates pulmonary fibrosis via CCL18. Am J Respir Crit Care Med. 2006;173(7):781-792. doi:10.1164/rccm.200509-15180C

12. Moin ASM, Sathyapalan T, Atkin SL, Butler AE. Pro-fibrotic M2 macrophage markers may increase the risk for COVID-19 in type 2 diabetes with obesity. Metabolism. 2020;112:154374. doi:10.1016/j. metabol.2020.154374

13. Mantovani A, Sica A, Sozzani S, Allavena P, Vecchi A, Locati M. The chemokine system in diverse forms of macrophage activation and polarization. Trends Immunol. 2004;25(12):677-686. doi:10.1016/j.it.2004.09.015

14. Cascella M, Rajnik M, Aleem A, Dulebohn S, Napoli R. Features, evaluation, and treatment of coronavirus (COVID-19). In: StatPearls. Treasure Island (FL): StatPearls Publishing; 2021.

15. Goudouris ES. Laboratory diagnosis of COVID-19. J Pediatr (Rio J). 2021;97(1):7-12. doi:10.1016/j.jped.2020.08.001 
16. Prokop M, Van Rees Vellinga T, Van Ufford H, et al. CO-RADS a categorical CT assessment scheme for patients suspected of having COVID-19 - definition and evaluation. Radiology. 2020;296 (2):97-104. doi:10.1148/radiol.2020201473

17. Li X, Ma X. Acute respiratory failure in COVID-19: is it "typical" ARDS? Crit Care. 2020;24(1):198. doi:10.1186/s13054-020-02911-9

18. Richardson S, Hirsch JS, Narasimhan M, et al. Presenting characteristics, comorbidities, and outcomes among 5700 patients hospitalized with COVID-19 in the New York city area. JAMA. 2020;323 (20):2052-2059. doi:10.1001/jama.2020.6775

19. Gong MN, Bajwa EK, Thompson BT, Christiani DC. Body mass index is associated with the development of acute respiratory distress syndrome. Thorax. 2010;65(1):44-50. doi:10.1136/thx.2009.117572

20. Misharin AV, Scott Budinger GR, Perlman H. The lung macrophage: a Jack of all trades. Am J Respir Crit Care Med. 2011;184 (5):497-498. doi:10.1164/rccm.201107-1343ED

21. Fernandez-Patron C, Kassiri Z, Leung D. Modulation of systemic metabolism by MMP-2: from MMP-2 deficiency in mice to MMP-2 deficiency in patients. Compr Physiol. 2016;6:1935-1949.

22. Elkington PT, Friedland JS. Matrix metalloproteinases in destructive pulmonary pathology. Thorax. 2006;61(3):259-266. doi:10.1136/ thx.2005.051979

23. Oikonomidi S, Kostikas K, Tsilioni I, Tanou K, Gourgoulianis KI, Kiropoulos TS. Matrix metalloproteinases in respiratory diseases: from pathogenesis to potential clinical implications. Curr. Med. Chem. 2009;16(10):1214-1228. doi:10.2174/092986709787846587

24. Hardy E, Fernandez-Patro C. Targeting MMP-regulation of inflammation to increase metabolic tolerance to COVID-19 pathologies: a hypothesis. Biomolecules. 2021;11(3):390. doi:10.3390/biom11030390

25. Hsu AT, Barrett CD, DeBusk MG, et al. Kinetics and role of plasma matrix metalloproteinase- 9 expression in acute lung injury and the acute respiratory distress syndrome. Shock. 2015;44(2):128-136. doi:10.1097/SHK.0000000000000386

26. Ueland T, Holter JC, Holten AR, et al. Distinct and early increase in circulating MMP-9 in COVID-19 patients with respiratory failure. $J$ Infect. 2020;81(3):41-43. doi:10.1016/j.jinf.2020.06.061
27. Siebler J, Galle PR, Weber MM. The gut liver axis: endotoxemia, inflammation, insulin resistance and NASH. J Hepatol. 2008;48 (6):1032-1034. doi:10.1016/j.jhep.2008.03.007

28. Weiss J. Bactericidal/permeability-increasing protein (BPI) and lipopolysaccharide - binding protein (LBP): structure, function and regulation in host defense against Gram-negative bacteria. Biochem Soc Trans. 2003;31(4):785-790. doi:10.1042/bst0310785

29. Ruiz AG, Casafont F, Crespo J, et al. Lipopolysaccharide-binding protein plasma levels and liver TNF- $\alpha$ gene expression in obese patients: evidence for the potential role of endotoxin in the pathogenesis of non-alcoholic steatohepatitis. Obes Surg. 2007;17 (10):1374-1380. doi:10.1007/s11695-007-9243-7

30. Yang X, Yu Y, Xu J, et al. Clinical course and outcomes of critically ill patients with SARS-CoV-2 pneumonia in Wuhan, China: a Single-Centered, Retrospective, Observational Study. Lancet Respir. 2020;8(5):475-481. doi:10.1016/S2213-2600(20)30079-5

31. Welker C, Huang J, Ramakrishna H. Acute respiratory distress syndrome update, with Coronavirus disease 2019 focus. J Cardiothoracic Vasc Anesth. 2021;1-8. doi:10.1053/j.jvca.2021.02.053

32. Xu W, Sun NN, Gao HN, et al. Risk factors analysis of COVID 19 patients with ARDS and prediction based on machine learning. Sci Rep. 2021;11(1):2933. doi:10.1038/s41598-021-82492-x

33. Varga J, Lafyatis R. Etiology and pathogenesis of systemic sclerosis. In: Rheumatology: Sixth Edition. Vol. 2. Elsevier Inc; 2015.

34. Petrey AC, Qeadan F, Middleton AE, Pinchuk IV, Campbell RA, Beswick EJ. Cytokine release syndrome in COVID-19: innate immune, vascular, and platelet pathogenic factors differ in severity of disease and sex. J Leukoc Biol. 2021;109(1):55-66. doi:10.1002/ JLB.3COVA0820-410RRR

35. Ferreira-Gomes M, Kruglov A, Durek P, Heinrich F. SARS-CoV-2 in severe COVID-19 induces a TGF- $\beta$-dominated chronic immune response that does not target itself. Nat Commun. 2021;12(1). doi:10.1038/s41467-021-22210-3
Infection and Drug Resistance

\section{Publish your work in this journal}

Infection and Drug Resistance is an international, peer-reviewed openaccess journal that focuses on the optimal treatment of infection (bacterial, fungal and viral) and the development and institution of preventive strategies to minimize the development and spread of resistance. The journal is specifically concerned with the epidemiology of

\section{Dovepress}

antibiotic resistance and the mechanisms of resistance development and diffusion in both hospitals and the community. The manuscript management system is completely online and includes a very quick and fair peerreview system, which is all easy to use. Visit http://www.dovepress.com/ testimonials.php to read real quotes from published authors. 